\title{
Application of the sea urchin embryo test in toxicity evaluation and effect directed analysis of wastewater treatment plant effluents
}

\section{Supplementary material}

Leire Mijangos ${ }^{a, b} *$, Martin Krauss ${ }^{d, e}$, Laura de Miguel ${ }^{b, c}$, Haizea Ziarrusta ${ }^{a, b}$, Maitane Olivares ${ }^{a, b}$, Olatz

Zuloaga $^{a, b}$, Urtzi Izaguirre ${ }^{b, c}$, Tobias Schulze ${ }^{\mathrm{d}, \mathrm{e}}$, Werner Brack $^{\mathrm{d}, \mathrm{e}}$, Ailette Prieto ${ }^{a, b}$, Nestor Etxebarria ${ }^{a, b}$

${ }^{a}$ Department of Analytical Chemistry, Faculty of Science and Technology, University of the Basque Country (UPV/EHU), P.O. Box 644, 48080 Bilbao, (Basque Country), Spain

${ }^{b}$ Research Centre for Experimental Marine Biology and Biotechnology (PIE), University of the Basque Country (UPV/EHU), Areatza z/g, 48620 Plentzia, (Basque Country), Spain

'Department of Zoology and Animal Cell Biology, University of the Basque Country (UPV-EHU), E-48080 Bilbao, Basque Country, Spain

d UFZ - Helmholtz Centre for Environmental Research, Permoserstr. 15, 04318 Leipzig, Germany

e Institute for Environmental Research, RWTH Aachen University, 52074 Aachen, Germany 
Supplementary Material 1 is formed by 22 pages which include the following information:

\section{SECTIONS}

S1. Reagents and materials

S2. Sampling

S3. Sea urchin Embryo Test

S4. EDA and fractionation

S5. Non-targeted analysis

S6. EDA-SET

S7. References

TABLES.

Table S1. Name, location, treatment, effluents discharge estuaries, water flow and influents sources of the WWTPs studied in this work.

Table S2. Water flow and effluent physicochemical parameters from Galindo, Gorliz and Mungia WWTPs.

Table S3. Fraction names, elution time windows and water content of the resulting fractions after the consecutive fractionation performed with two columns (Nucleodur $\mathrm{C}_{18}$ gravity and Imtakt aminopropyl).

Table S4. Compound Discoverer (2.1) workflow settings and parameters.

Table S5. Concentrations of each of the 6 targeted compounds (mexacarbate, albendazole, mebendazole, paroxetine, amitriptyline and fenpropidin) in the artificial mixture. Concentrations of REF1 is based on the concentrations presented in the raw sample at equal REF

\section{FIGURES}

Figure S1. Schematic representation of the experimental design of the effect-directed analysis approach. $\Sigma F$, recombined fractions; AP fract., fractionation with aminopropyl column; C18 fract., fractionation with C18 column; REF, relative enrichment factor; SET, sea urchin embryo toxicity; SPE, solid phase extraction; TU, toxic unit.

Figure S2. Compound Discoverer (2.1) workflow

Figure S3. Types of embryonic stages and developmental abnormalities of sea urchin Paracentrotus lividus observed in this study after $48 \mathrm{~h}$ incubation period. a) normal 4 arm pluteus stage (level 0); b) crossed tip (level 1); c) fused arms (level 1); d)separated tip (level 1); e) incomplete skeletal rods (level 2); f) absence of skeletal rods (level 2); g) folded tip (level 2); h) pre-pluteus stage; i) Undeveloped stage.

Figure S4. The log dose-response curves of the active samples (Ga2 raw, RC18, F113, RAP and F24) obtained with a) size increase end-point and b) skeleton malformation end-point. Straight lines show the EC fit values obtained with Probit and dashed line the confidence level (95\%). 
Figure S5. MS ${ }^{2}$ spectra (HCD 10, 35 and 75) of a) albendazole, b) mebendazole, c) amitriptyline, d) fenpropidin e) paroxetine and d) fragments explanation of two potential candidates (mexacarbate and neostigmine) which match with the precursor ion. Only the mayor fragments have been included (rounded).

Figure S6. The log dose-response curves of the identified chemicals tested individually: a) paroxetine, b) albendazole, c) mebendazole, d) mexacarbate, e) amitriptyline and f) fenpropidin. Straight lines show the EC fit values obtained with Probit and dashed line the confidence level (95\%).

Figure S7. The log dose-response curves of the artificial mixture of the identified 6 chemicals tested all together. Straight lines show the EC fit values obtained with Probit and dashed line the confidence level (95\%). 


\section{S1. Reagents and materials}

Names, use, CAS numbers, molecular formulas and brand target compounds are summarized in S1 supplementary material (SM) 2. All the reference standards used in this study have a purity of at least $97 \%$.

Oasis hydrophilic-lipophilic balanced (HLB) 200 mg-SPE cartridges were purchased from Waters (Milford, USA). Bond-Elut Plexa and Strata X-AW bulk sorbents used in the effect-directed analysis approach were purchased to Agilent (Santa Clara, CA, USA) and Phenomenex (Torrance, CA, USA), respectively. Empty solid phase extraction (SPE) tubes $(6 \mathrm{~mL}$ and $20 \mathrm{~mL}$ ) and polypropylene (PP) frits were purchased from Supelco (Bellefonte, PA, USA).

Formic acid (> 98\%), ethylenediaminetetraacetic sodium salt (EDTA $\geq 99.9 \%)$ and sodium thiosulfate $(\geq 98)$ were supplied by Panreac (Barcelona, Spain). Methanol (MeOH, HPLC grade, 99.9\%), ethyl acetate (EtOAC, HPLC grade, 99.9\%), acetone (HPLC grade, 99.9\%) and ammonium solution (25\% as $\mathrm{NH}_{3}$ ) used in the SPE extraction were obtained from Sigma Aldrich (St. Louis, MO, USA). Ultra-pure water was obtained using a Milli-Q water purification system ( $<0.05 \mathrm{~S} / \mathrm{cm}$, Milli-Q model 185, Millipore, Bedford, MA, USA). Dimethyl sulfoxide (DMSO, cell culture grade) used in the bioassays was supplied by Panreac.

LC-MS grade $\mathrm{MeOH}$, water and formic acid (Optima grade) purchased from Sigma Aldrich were used as mobile phase in the fractionation, whereas Optima grade water, acetonitrile, isopropanol and formic acid provided by Fischer Scientific (Geel, Belgium) were used as mobile phase in the LC-HRMS. 


\section{S2. Sampling.}

Table S1. Name, location, treatment, effluents discharge estuaries, water flow and influents sources of the WWTPs studied in this work.

\begin{tabular}{|c|c|c|c|c|c|}
\hline WWTP & Coordinates & Treatment & Effluents discharge estuaries & $\begin{array}{c}\text { Median } \\
\text { water flow } \\
\text { (m³/day) }\end{array}$ & Influent sources \\
\hline Galindo & $\begin{array}{l}-2.97103 \mathrm{~W} \\
43.28796 \mathrm{~N}\end{array}$ & $\begin{array}{c}2^{\text {nd }} \\
(\mathrm{Ga} 2) \\
3^{\text {rd }} \\
\text { chlorination } \\
(\mathrm{Ga} 3)\end{array}$ & Bilbao estuary & $1.0 \mathrm{e} 9$ & $\begin{array}{c}\text { Industrial 3.2\%, } \\
\text { Hospital 0.5\%, } \\
\text { Domestic } 96.3 \% \\
\text { (> } 1000000 \text { inhabitant) }\end{array}$ \\
\hline Gorliz & $\begin{array}{l}-2.94244 \mathrm{~W} \\
43.41229 \mathrm{~N}\end{array}$ & $2^{\text {nd }}$ & $\begin{array}{l}\text { Plentzia estuary } \\
\text { It releases the effluent into the estuary mouth } \\
\text { through a submarine pipe located to } 1000 \mathrm{~m} \\
\text { from the coast with an } 18 \mathrm{~m} \text { depth. }\end{array}$ & $1.4 \mathrm{e} 6$ & $\begin{array}{c}\text { Industrial } 0 \% \text {, } \\
\text { Hospital } 1.3 \% \\
\text { Domestic } 98.7 \% \\
\text { (10600 inhabitants) }\end{array}$ \\
\hline Mungia & & $2^{\text {nd }}$ & $\begin{array}{c}\text { Plentzia estuary } \\
\text { It releases the effluent into the upper part } \\
\text { (22 km with to respect the mouth) of Plentzia } \\
\text { estuary }\end{array}$ & $5.4 \mathrm{e} 3$ & $\begin{array}{c}\text { Industrial } 3.1 \% \text {, } \\
\text { Hospital } 0 \%, \\
\text { Domestic } 96.9 \% \\
\text { (17000 inhabitants) }\end{array}$ \\
\hline Gernika & $\begin{array}{l}-2.6739 \mathrm{~W} \\
43.3239 \mathrm{~N}\end{array}$ & $1^{\mathrm{st}}$ & $\begin{array}{l}\text { Urdaibai estuary. } \\
\text { It discharges directly to the estuary of } \\
\text { Urdaibai, which is declared Reserve of The } \\
\text { Biosphere by Unesco since } 1984 .\end{array}$ & $-b$ & $\begin{array}{l}\text { Industrial 25.33\%, } \\
\text { Hospital } 0.2 \% \text {, } \\
\text { Domestic } 74.46 \% \\
\text { (70000 inhabitants) }\end{array}$ \\
\hline
\end{tabular}

a) Currently, it is only for private use in the WWTP

b) Unknown (but $<10 \%$ of the total flow)

Table S2. Water flow and effluent physicochemical parameters from Galindo, Gorliz and Mungia WWTPS

\begin{tabular}{ccccccccccc}
\hline WWTP & $\begin{array}{c}\text { Flow } \\
\mathrm{m}^{3} / \text { day }\end{array}$ & $\mathrm{pH}$ & $\begin{array}{c}\mathrm{TSS} \\
\mathrm{mg} / \mathrm{L}\end{array}$ & $\begin{array}{c}\mathrm{VSS} \\
\mathrm{mL} / \mathrm{L}\end{array}$ & $\begin{array}{c}\mathrm{COD} \\
\mathrm{mg} / \mathrm{L}\end{array}$ & $\begin{array}{c}\mathrm{BOD} \\
\mathrm{mg} / \mathrm{L}\end{array}$ & $\begin{array}{c}\mathrm{NH}_{3} \\
\mathrm{mg} / \mathrm{L}\end{array}$ & $\begin{array}{c}\mathrm{NO}_{3}{ }^{-} \\
\mathrm{mg} / \mathrm{L}\end{array}$ & $\begin{array}{c}\mathrm{PO}_{4}{ }^{3-} \\
\mathrm{mg} / \mathrm{L}\end{array}$ & $\begin{array}{c}\mathrm{Conductivity} \\
(\mu \mathrm{S} / \mathrm{CM})\end{array}$ \\
\hline Galindo & 305572 & 7.10 & $<6$ & 4.00 & 37.00 & $<4$ & $<0,50$ & 7.42 & 3.89 & $3,670.00$ \\
Gorliz & 3404 & 7.40 & 16.00 & $<3$ & 51.00 & 4.80 & 7.51 & 12.70 & 2.88 & $1,270.00$ \\
Mungia & 6161 & 7.50 & 6.00 & $<3$ & 43.00 & $<4$ & 0.58 & 0.64 & 0.53 & 531.00 \\
\hline
\end{tabular}

TSS: total suspended solid; VSS: volatile suspended solids, COD: chemical oxygen demand; BOD: Biological oxygen demand after 5 days; 


\section{S3. Sea urchin Embryo Test (SET)}

Adults of sea urchins ( $P$ lividus) were provided by the ECIMAT (Galicia, Spain) or collected from an intertidal area of Armintza $(43.43347 \mathrm{~N}, 2.89889 \mathrm{~W}$, Basque Country) and maintained in aquaria at the Plentzia Marine Station (PiE). Seawater tanks were maintained at $15 \pm 1^{\circ} \mathrm{C}$ and natural photoperiod. Every two days sea urchins were fed with macroalgae and dregs were siphoned.

Gametes were obtained by osmotic-shock-induced spawning injecting $1 \mathrm{~mL}$ of potassium chloride $(\mathrm{KCl}$, $0.5 \mathrm{~mol} / \mathrm{L})$ through the peri-oral membrane into coelom ${ }^{1}$. Afterwards, gametes were observed under a microscope to check their viability (eggs roundness and sperm mobility) and the viable ones were pooled.

The fertilisation procedure was carried out as described by Fernández and Beiras ${ }^{2}$. A dense suspension of oocytes in control FSW was fertilised with a few $\mu \mathrm{L}$ of non-diluted sperm. $20 \mu \mathrm{L}$-aliquots $(n=4)$ were taken to record fertilisation success (assessed by the percentage of eggs showing a fertilisation membrane) and egg density through an inverted microscope (Nikon eclipse Ti-S). Eggs were counted using a Sedgewickrafter counting cell (Pyser Optics, Edenbridge, United Kingdom). Within 30 minutes, the fertilised egg suspension was distributed in glass vials $(20 \mathrm{~mL})$ containing a known volume of test sample $(3 \mathrm{~mL})$, assuring a final concentration of 40 eggs $/ \mathrm{mL}$. Afterwards, fertilized sea urchin embryo egg were added to test samples and placed in an incubator at $20^{\circ} \mathrm{C}$ for $48 \mathrm{~h}$ in darkness until larvae reach the four arm-pluteus stage. After the incubation, larvae were preserved by adding a one drop per sample of $40 \%$ formalin. 


\section{S4. EDA and fractionation}

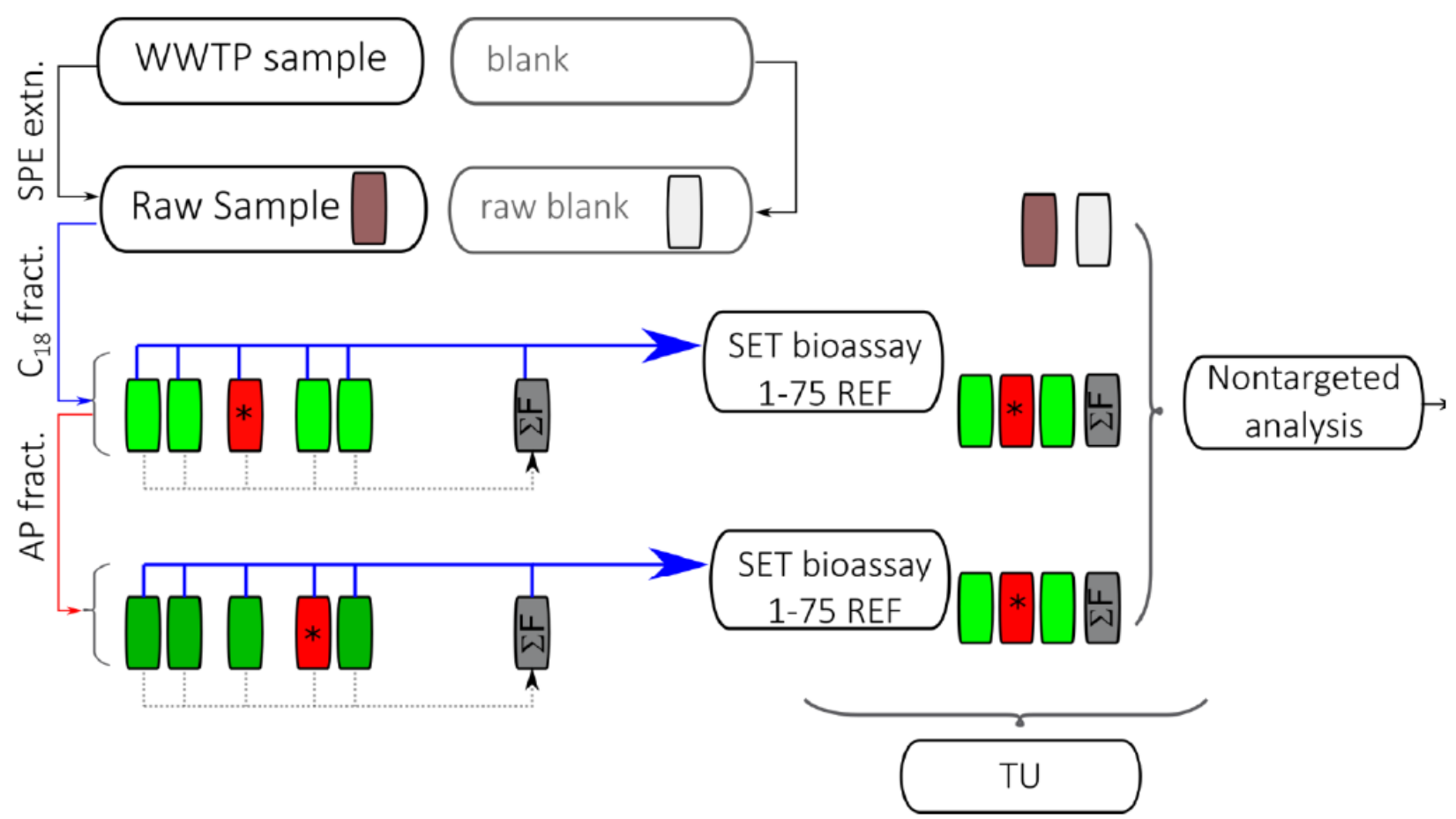

Figure S1. Schematic representation of the experimental design of the effect-directed analysis approach. $\Sigma \mathrm{F}$, recombined fractions; AP fract., fractionation with aminopropyl column; $\mathrm{C}_{18}$ fract., fractionation with $\mathrm{C}_{18}$ column; REF, relative enrichment factor; SET, sea urchin embryo toxicity; SPE, solid phase extraction; TU, toxic unit. 
Table S3. Fraction names, elution time windows and water content of the resulting fractions after the consecutive fractionation performed with two columns (Nucleodur $\mathrm{C}_{18}$ gravity and Imtakt aminopropyl).

\begin{tabular}{|c|c|c|c|c|c|c|}
\hline \multirow{4}{*}{ Fraction } & \multicolumn{6}{|c|}{ Fractionation approaches } \\
\hline & \multicolumn{3}{|c|}{$1^{\text {st }}$ fractionation step } & \multicolumn{3}{|c|}{$2^{\text {nd }}$ fractionation step } \\
\hline & \multicolumn{3}{|c|}{ Nucleodur $\mathrm{C}_{18}$ Gravity column } & \multicolumn{3}{|c|}{ Imtakt aminopropylcolumn } \\
\hline & Name & $\begin{array}{c}\text { Fraction } \mathrm{RT}^{\mathrm{a}} \\
\text { (min) }\end{array}$ & $\begin{array}{c}\text { Water content } \\
\text { (\%) }\end{array}$ & Name & $\begin{array}{c}\text { Fraction } \mathrm{RT}^{\mathrm{a}} \\
\text { (min) }\end{array}$ & $\begin{array}{c}\text { Water content } \\
\text { (\%) }\end{array}$ \\
\hline 1 & F1 & $0-2$ & 70 & F13-1 & $0-3$ & 92 \\
\hline 2 & F2 & $2-4$ & 70 & F13-2 & $3-6$ & 84 \\
\hline 3 & F3 & $4-6$ & 68 & F13-3 & $6-9$ & 77 \\
\hline 4 & $\mathrm{~F} 4$ & $6-8$ & 64 & F13-4 & $9-12$ & 69 \\
\hline 5 & F5 & $8-10$ & 60 & F13-5 & $12-15$ & 61 \\
\hline 6 & F6 & $10-12$ & 56 & F13-6 & $15-18$ & 53 \\
\hline 7 & F7 & $12-14$ & 52 & F13-7 & $18-21$ & 45 \\
\hline 8 & F8 & $14-16$ & 48 & F13-8 & $21-24$ & 37 \\
\hline 9 & F9 & $16-18$ & 44 & F13-9 & $24-27$ & 29 \\
\hline 10 & F10 & $18-20$ & 40 & F13-10 & $27-30$ & 21 \\
\hline 11 & F11 & $20-22$ & 36 & F13-11 & $30-33$ & 13 \\
\hline 12 & F12 & $22-24$ & 32 & F13-12 & $33-36$ & 6 \\
\hline 13 & F13 & $24-26$ & 28 & F13-13 & $36-39$ & 5 \\
\hline 14 & F14 & $26-28$ & 24 & F13-14 & $39-42$ & 5 \\
\hline 15 & F15 & $28-30$ & 20 & F13-15 & $42-45$ & 5 \\
\hline 16 & F16 & $30-32$ & 16 & & & \\
\hline 17 & F17 & $32-34$ & 12 & & & \\
\hline 18 & F18 & $34-36$ & 10 & & & \\
\hline 19 & F19 & $36-39$ & 5 & & & \\
\hline 20 & $\mathrm{~F} 20$ & $39-42$ & 5 & & & \\
\hline 21 & $F 21$ & $42-50$ & 5 & & & \\
\hline
\end{tabular}

\footnotetext{
${ }^{a}$ The fraction collector was started with a delay of 4 min.
} 


\section{S5. Non-targeted analysis}

The analysis were performed in a Thermo Scientific Dionex UltiMate 3000 UHPLC coupled to a Thermo Scientific Q Exactive quadrupole-Orbitrap mass spectrometer equipped with a heated ESI source (HESI, Thermo, CA, USA).

The separation was carried out at $0.3 \mathrm{~mL} / \mathrm{min}$ and $35^{\circ} \mathrm{C}$ of flow rate and temperature, respectively, on an ACE UltraCore 2.5 SuperPhenylhexyl $(2.1 \mathrm{mmx} 100 \mathrm{~mm}, 2.5 \mu \mathrm{m}$ ) column coupled to a pre filter (Vivi Jour, Schenkon, Suitzlerdan) from Waters (Milford, Massachusetts, United States). Milli-Q water was used as mobile phase $\mathrm{A}$ and acetonitrile as mobile phase $\mathrm{B}$, both containing $0.1 \%$ formic acid. The injection volume was set to $5 \mu \mathrm{L}$. The eluent gradient profile was as follows: linear change of $85 \%$ A to $70 \%$ up to $4 \mathrm{~min}$, another linear change to 50\% A up to 4 min (hold $12 \mathrm{~min}$ ), another linear change to $10 \% \mathrm{~A}$ up to $10 \mathrm{~min}$ (hold $15 \mathrm{~min}$ ) and a final linear change to $85 \%$ A up to $3 \mathrm{~min}$. Lastly, 5 min to regain initial conditions.

The Orbitrap was operated in the corresponding ionization mode in full scan - data dependant MS $^{2}$ (Full MS-ddMS ${ }^{2}$ ) discovery acquisition mode. One full scan at a resolution of 70,000 full width at half maximum (FWHM) at m/z 200 over a scan range of m/z 70-1000 was followed by three ddMS ${ }^{2}$ scans at a resolution of $17,500 \mathrm{FWHM}$ at $\mathrm{m} / \mathrm{z} 200$, with an isolation window of $0.8 \mathrm{Da}$. The stepped normalized collision energy (NCE) in the higher-energy collisional dissociation (HCD) cell was set to 10, 35 and $75 \mathrm{eV}$. Negative and positive voltages were measured in different injections runs. The HESI source parameters in positive mode were set to $3.2 \mathrm{kV}$ spray voltage, $300{ }^{\circ} \mathrm{C}$ capillary temperature, 35 arbitrary units (au) sheath gas (nitrogen), 10 au auxiliary gas, 1 au sweep gas, $280{ }^{\circ} \mathrm{C}$ auxiliary gas heater and S-lens RF level 55.0. The HESI source parameters in negative mode were set to $3.2 \mathrm{kV}$ spray voltage, $330^{\circ} \mathrm{C}$ capillary temperature, 48 au sheath gas, 11 au auxiliary gas, 2 au sweep gas, $310^{\circ} \mathrm{C}$ auxiliary gas heater and S-lens RF level 55.0. External calibration of the instrument was conducted immediately prior to analysis using Pierce LTQ ESI Calibration Solutions (Thermo Scientific, Waltham, Massachusetts, United States). The instrument was controlled by Xcalibur 4.0 software (Thermo).

Data analysis was done using Compound Discoverer 2.1 (CD; Thermo-Fisher Scientific). The workflow and settings used for the data evaluation are summarized in Figure S2 and Table S4 in SM1 


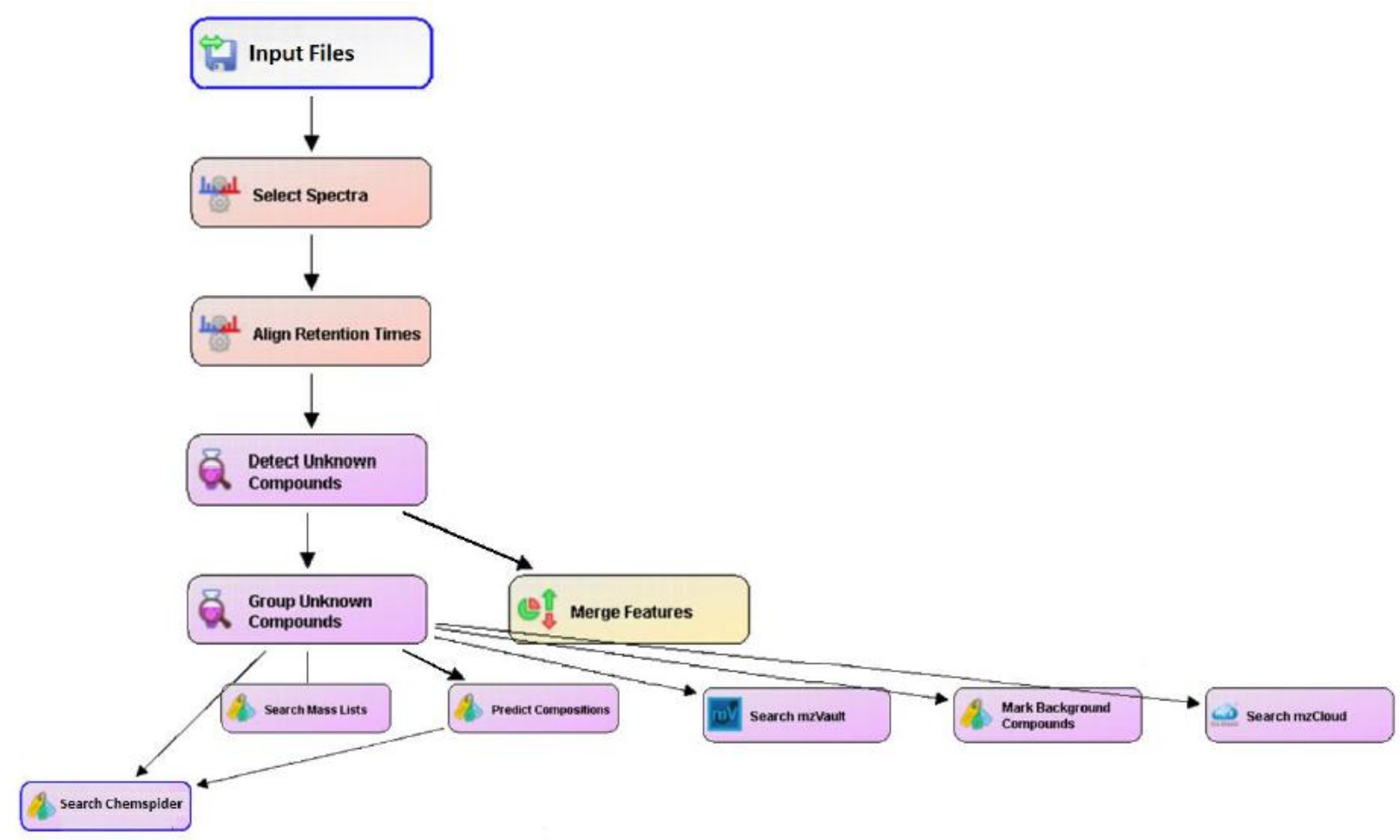

Figure S2 Compound Discoverer (2.1) workflow

Table S4. Compound Discoverer (2.1) workflow settings and parameters

\begin{tabular}{ll} 
1. Select Spectra $\quad$ 1.1 General settings & - Precursor Selection: Use MS (N - 1) Precursor \\
& - Use New Precursor Reevaluation: True \\
& - Use Isotope Pattern in Precursor Reevaluation: True \\
& - Store Chromatograms: False \\
\hline 1.2 Spectrum properties Filter & - Lower RT Limit: 0 \\
& - Upper RT Limit: 0 \\
& - First Scan: 0 \\
& - Last Scan: 0 \\
& - Ignore Specified Scans: (not Specified) \\
& - Lowest Charge State: 0 \\
& - Highest Charge State: 0 \\
& - Min. Precursor Mass: $100 \mathrm{Da}$ \\
& - Max. Precursor Mass: $5000 \mathrm{Da}$ \\
& - Total Intensity Threshold: 0 \\
& - Minimum Peak Count: 1
\end{tabular}


Table S4. Compound Discoverer (2.1) workflow settings and parameters

\begin{tabular}{|c|c|c|}
\hline \multirow{3}{*}{ 1. Select Spectra } & 1.3 Scan event Filters & $\begin{array}{l}\text { - Mass Analyzer: (not Specified) } \\
\text { - MS Order: Any } \\
\text { - Activation Type: (not Specified) } \\
\text { - Min. Collision Energy: } 0 \\
\text { - Max. Collision Energy: } 1000 \\
\text { - Scan Type: Any } \\
\text { - Polarity Mode: (not Specified) }\end{array}$ \\
\hline & 1.4 peak filters & - S/N Threshold (FT-only): 1.5 \\
\hline & $\begin{array}{l}\text { 1.5. Replacements for } \\
\text { Unrecognized Properties }\end{array}$ & $\begin{array}{l}\text { - Unrecognized Charge Replacements: } 1 \\
\text { - Unrecognized Mass Analyser Replacements: ITMS } \\
\text { - Unrecognized MS Order Replacements: MS }{ }^{2} \\
\text { - Unrecognized Activation Type Replacements: CID } \\
\text { - Unrecognized Polarity Replacements: + } \\
\text { - Unrecognized MS Resolution@200 Replacements: } 60000 \\
\text { - Unrecognized MSn Resolution@200 Replacements: } 30000\end{array}$ \\
\hline $\begin{array}{l}\text { 2. Align Retention } \\
\text { times }\end{array}$ & 2.1. General Settings & $\begin{array}{l}\text { - Alignment Model: Adaptive curve } \\
\text { - Alignment Fallback: Use Linear Model } \\
\text { - Maximum Shift [min]: } 2 \\
\text { - Shift Reference File: True } \\
\text { - Mass Tolerance: } 5 \text { ppm } \\
\text { - Remove Outlier: True }\end{array}$ \\
\hline \multirow[t]{2}{*}{$\begin{array}{l}\text { 3. Detect Unknown } \\
\text { Compounds }\end{array}$} & 3.1. General Settings & $\begin{array}{l}\text { - Mass Tolerance [ppm]: } 5 \text { ppm } \\
\text { - Intensity Tolerance [\%]: } 30 \\
\text { - S/N Threshold: } 3 \\
\text { - Min. Peak Intensity: } 500000 \\
\text { - Ions: [M+Cl]-1; [M+FA-H]-1; [M+H]+1; [M+H+MeOH]+1; [M+K]+1; } \\
\text { [M+Na]+1; [M-H]-1; [M-H-H2O]-1 } \\
\text { - Base lons: [M+H]+1; [M-H]-1 } \\
\text { - Min. Element Counts: CH } \\
\text { - Max. Element Counts: C90 H190 Br3 Cl4 F20 K2 N10 Na2 O18 P3 S5 }\end{array}$ \\
\hline & 3.2. Peak Detection & $\begin{array}{l}\text { - Filter Peaks: True } \\
\text { - Max. Peak Width [min]: } 0.8 \\
\text { - Remove Singlets: True } \\
\text { - Min. \# Scans per Peak: } 3 \\
\text { - Min. \# Isotopes: } 1\end{array}$ \\
\hline 4. Merge Features & 4.1 Peak consolidation & $\begin{array}{l}\text {-mass tolerance: } 5 \mathrm{ppm} \\
\text { - RT Tolerance } 0.1 \mathrm{~min}\end{array}$ \\
\hline \multirow[t]{2}{*}{$\begin{array}{l}\text { 5. Group Unknown } \\
\text { Compounds }\end{array}$} & 5.1. Compound Consolidation & $\begin{array}{l}\text { - Mass Tolerance: } 5 \text { ppm } \\
\text { - RT Tolerance [min]: } 0.5\end{array}$ \\
\hline & 5.2. Fragment Data Selection & - Preferred lons: $[\mathrm{M}+\mathrm{H}]+1 ;[\mathrm{M}-\mathrm{H}]-1$ \\
\hline \multirow[t]{2}{*}{ ChemSpider } & 6.1. Search Settings & $\begin{array}{l}\text { Database(s): ACToR: Aggregated Computational Toxicology Resource; } \\
\text { DrugBank; EAWAG BIOcatalysis/Biodegradation Databse; EPA DSSTox; } \\
\text { EPA Toxcast; FDA UNII-NLMBioCyc; KEGG; Mass Bank } \\
\text { - Mass Tolerance: } 5 \text { ppm } \\
\text { - Max. \# of results per compound: } 100 \\
\text { - Max. \# of Predicted Compositions to be searched per Compound: } 3 \\
\text { - Result Order (for Max. \# of results per compound): Order By } \\
\text { Reference Count (DESC) }\end{array}$ \\
\hline & 6.2. Predict Composition & - Check All Predicted Compositions: True \\
\hline
\end{tabular}


Table S4. Compound Discoverer (2.1) workflow settings and parameters

\begin{tabular}{|c|c|c|}
\hline 7. Search Mass Lists & 7.1. Search Settings & $\begin{array}{l}\text { - Input file(s): \EFS HRAM Compound Database_OZZ.csv } \\
\text { - Show extra Fields as Columns: False } \\
\text { - Consider Retention Time: True } \\
\text { - RT Tolerance : } 0.5 \\
\text { - Mass Tolerance: } 5 \text { ppm }\end{array}$ \\
\hline $\begin{array}{l}\text { 8.Predict } \\
\text { Composition }\end{array}$ & 8.1. Prediction Settings & $\begin{array}{l}\text { Mass Tolerance: } 5 \text { ppm } \\
\text { - Min. Element Counts: CH } \\
\text { - Max. Element Counts: C90 H190 Br3 Cl4 F20 K2 N10 Na2 O18 P3 S5 } \\
\text { - Min. RDBE: } 0 \\
\text { - Max. RDBE: } 40 \\
\text { - Min. H/C: } 0.1 \\
\text { - Max. H/C: } 3.5 \\
\text { - Max. \# Candidates: } 10 \\
\text { - Max. \# Internal Candidates: } 200\end{array}$ \\
\hline & 8.2. Pattern Matching & $\begin{array}{l}\text { Intensity Tolerance [\%]: } 30 \\
\text { - Intensity Threshold [\%]: } 0.1 \\
\text { - S/N Threshold: } 3 \\
\text { - Min. Spectral Fit [\%]: } 30 \\
\text { - Min. Pattern Cov. [\%]: } 80 \\
\text { - Use Dynamic Recalibration: True }\end{array}$ \\
\hline & 8.3. Fragments Matching & $\begin{array}{l}\text { - Use Fragments Matching: True } \\
\text { - Mass Tolerance: } 5 \text { ppm } \\
\text { - S/N Threshold: } 3\end{array}$ \\
\hline 9. Seach mzVault & 9.1 Seach settings & $\begin{array}{l}\text { - mzVault Library: ImzVault February 2017.db } \\
\text { - Compound Classes: All } \\
\text { - Match Ion Activation Type: True } \\
\text { - Match Ion Activation Energy: Match with Tolerance } \\
\text { - Ion Activation Energy tolerance: } 20 \\
\text { - Match Ionization Method: True } \\
\text { - Apply Intensity Method: true } \\
\text { - Remove precursor lon: true } \\
\text { - Precursor Mass Tolerance: } 10 \text { ppm } \\
\text { - FT Fragment Mass Tolerance: } 10 \text { ppm } \\
\text { - IT Fragment mass tolerance: } 0.4 \text { Da } \\
\text { - Match Analyzer Type: True } \\
\text { - Search Algorithm: HighChem HighRes } \\
\text { - Match factor Threshold: } 50 \\
\text { - Max. \# results: } 10\end{array}$ \\
\hline $\begin{array}{l}\text { 10. Mark } \\
\text { BackGround } \\
\text { compounds }\end{array}$ & 10.1 Seach settings & $\begin{array}{l}\text { - Max. Sample/Blank: } 5 \\
\text { - Max Max. Blank/Sample: } 0 \\
\text { - Hide Background: True }\end{array}$ \\
\hline
\end{tabular}


Table S4. Compound Discoverer (2.1) workflow settings and parameters

\begin{tabular}{|c|c|c|}
\hline 11. Search mzCloud & 11.1. Search Settings & $\begin{array}{l}\text { - Compound Classes: All } \\
\text { - Match Ion Activation Type: True } \\
\text { - Match Ion Activation Energy: Match with Tolerance } \\
\text { - Ion Activation Energy Tolerance: } 20 \\
\text { - Apply intensity threshold: True } \\
\text { - Precursor Mass Tolerance: } 10 \text { ppm } \\
\text { - FT Fragment Mass Tolerance: } 10 \text { ppm } \\
\text { - IT Fragment Mass Tolerance: } 0.4 \text { Da } \\
\text { - Search Algorithm: Cosine } \\
\text { - Similarity Search: Similarity Forward } \\
\text {--Library: Reference } \\
\text { - Post Processing: Recalibrated } \\
\text { - Match factor threshold: } 50 \\
\text { - Max. \# results per compound and spectrum: } 20\end{array}$ \\
\hline
\end{tabular}

\section{S6. EDA-SET}

Figure S3a-I in SM1 show representative malformations observed for the tested effluents in this work and the Figure S4 the modelled dose-response curves for the identified toxic samples (Raw, $R_{C 18}, F 13, R_{A P}$ and F13-4) in EDA approach. Figure S5a-e shows the $\mathrm{MS}^{2}$ spectra of albendazole, mebendazoleamitriptyline, fenpropidin and paroxetine, respectively. 

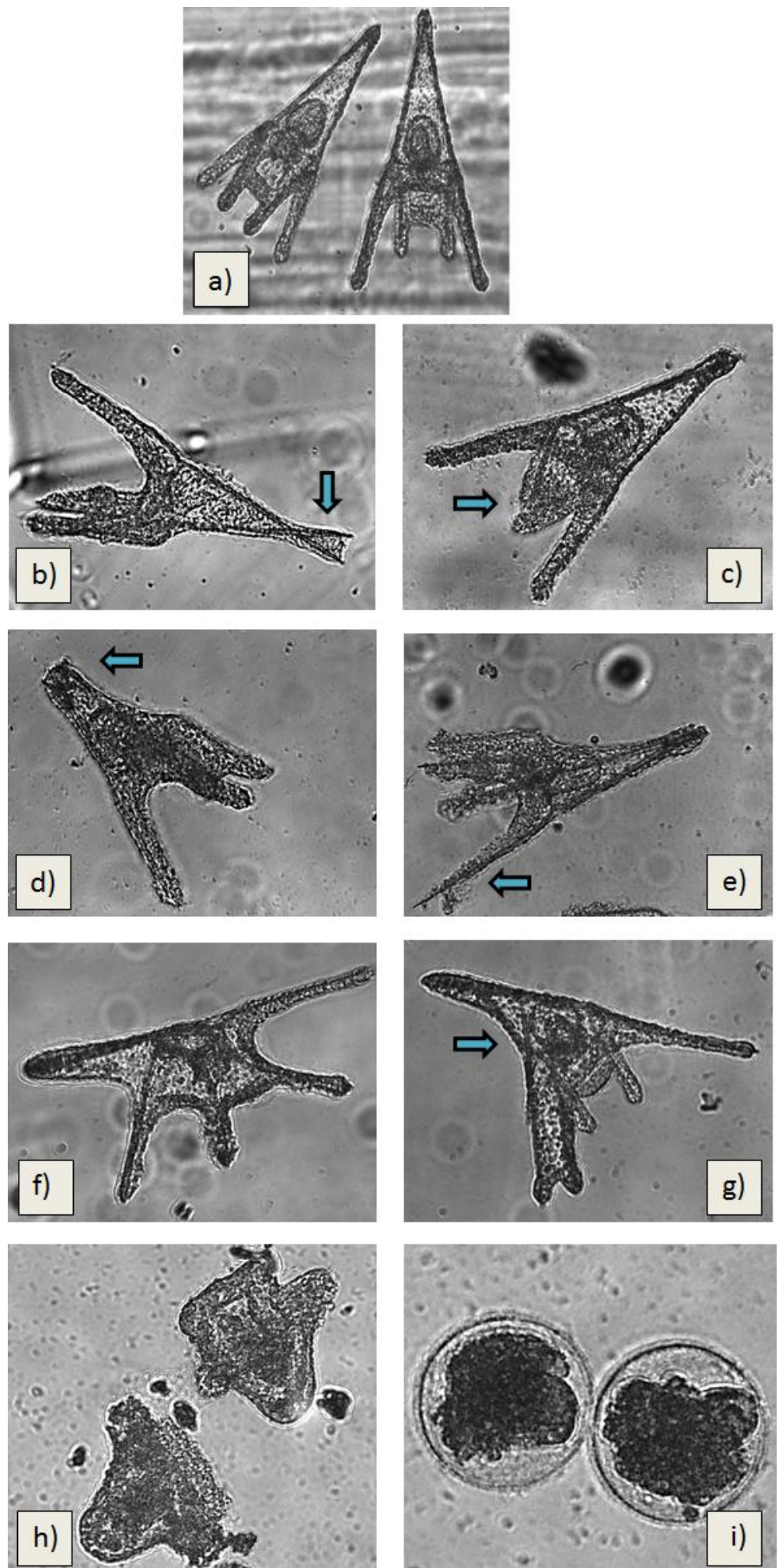

Figure S3 Types of embryonic stages and developmental abnormalities of sea urchin Paracentrotus lividus observed in this study after $48 \mathrm{~h}$ incubation period. a) normal 4 arm pluteus stage (level 0); b) crossed tip (level 1); c) fused arms (level 1); d)separated tip (level 1); e) incomplete skeletal rods (level 2); f) absence of skeletal rods (level 2); g) folded tip (level 2); h) pre-pluteus stage; i) Undeveloped stage. 

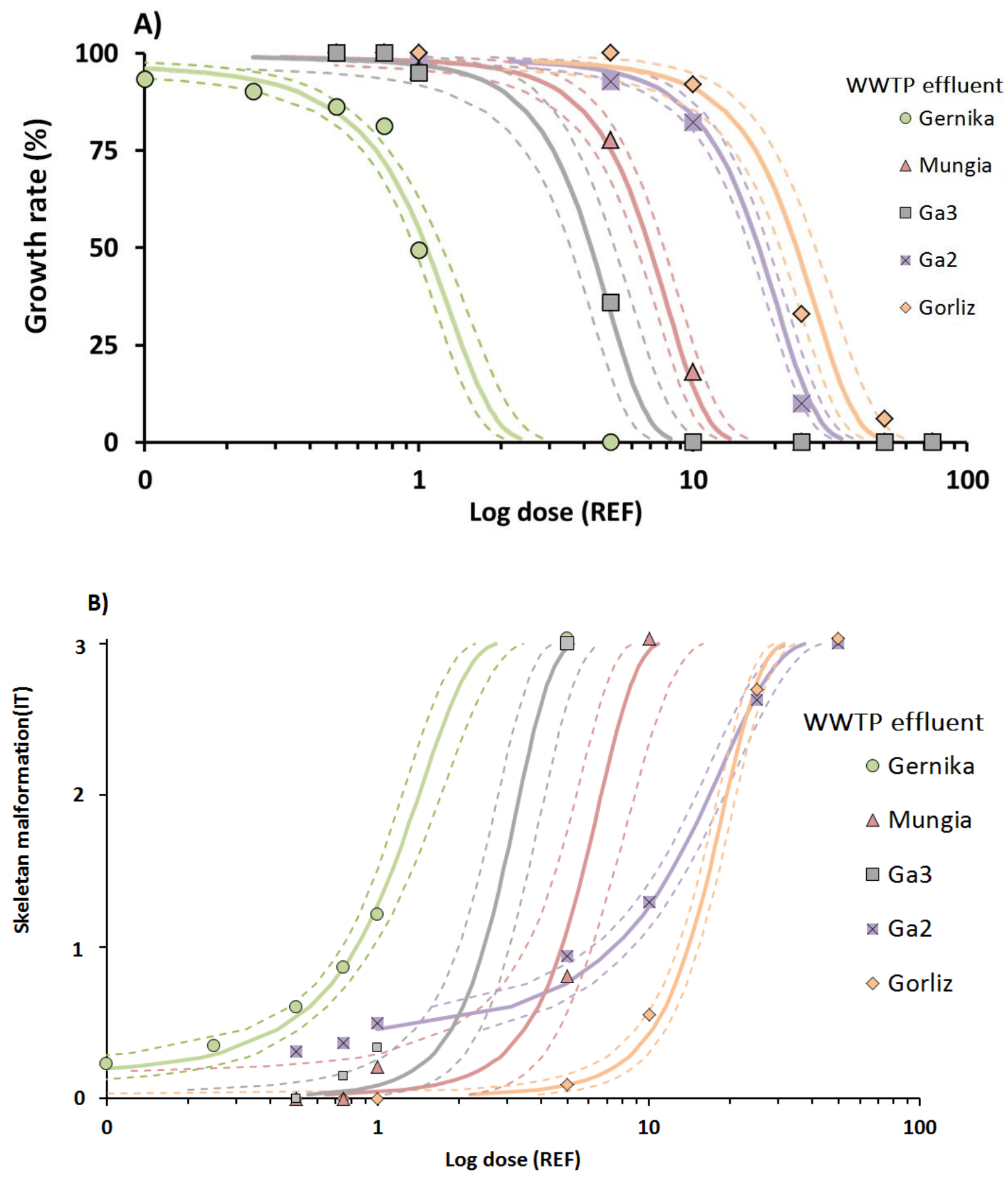

Figure S4. The log dose-response curves of the active samples ( $G a 2$ raw, $R_{C 18}, F 1_{13}, R_{A P}$ and $F 2_{4}$ ) obtained with a) size increase endpoint and b) skeleton malformation end-point. Straight lines show the EC fit values obtained with Probit and dashed line the confidence level (95\%). For interpretation of colored legend in these figures, the reader is referred to the web version of this article. 
A)
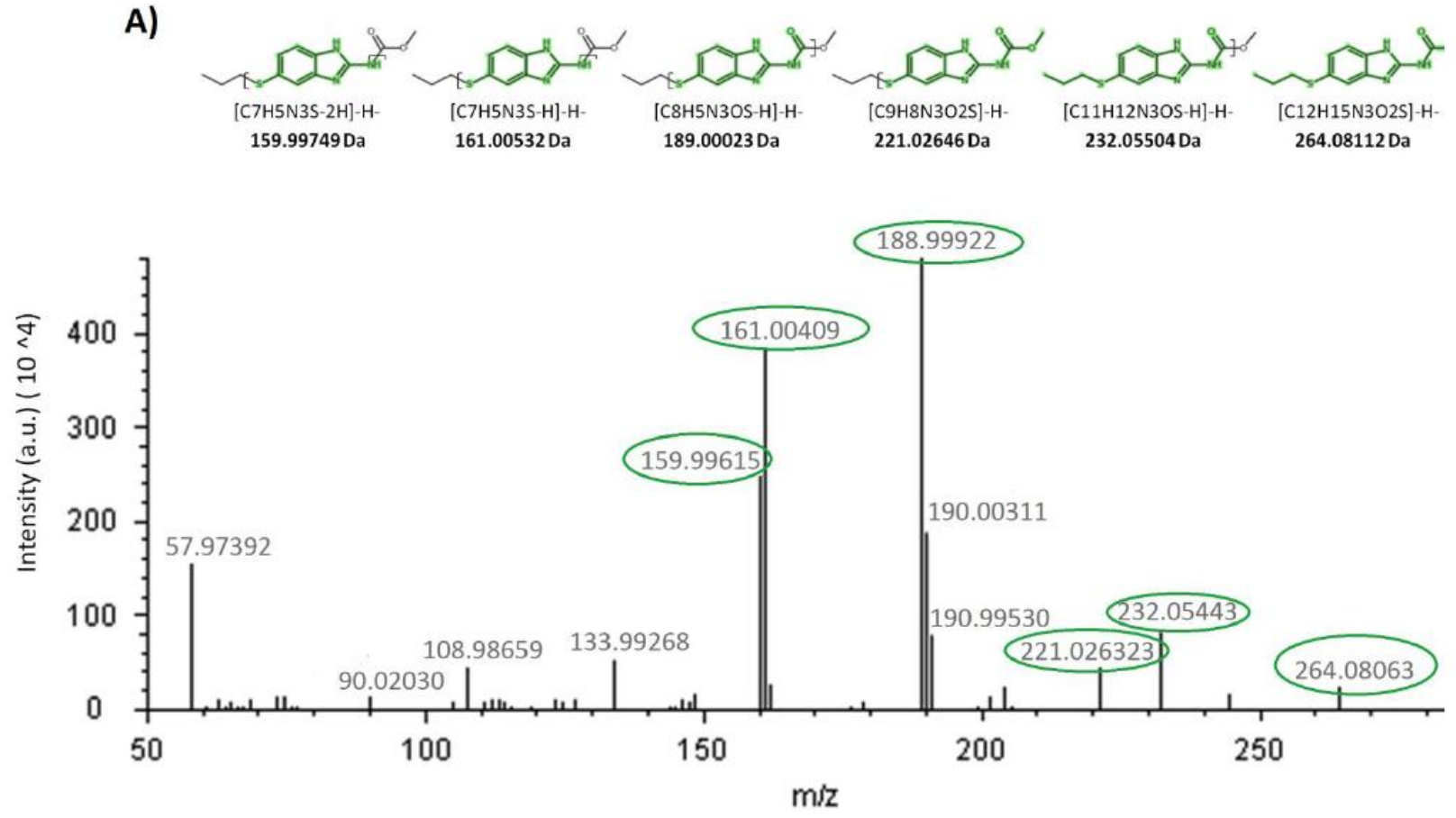

B)
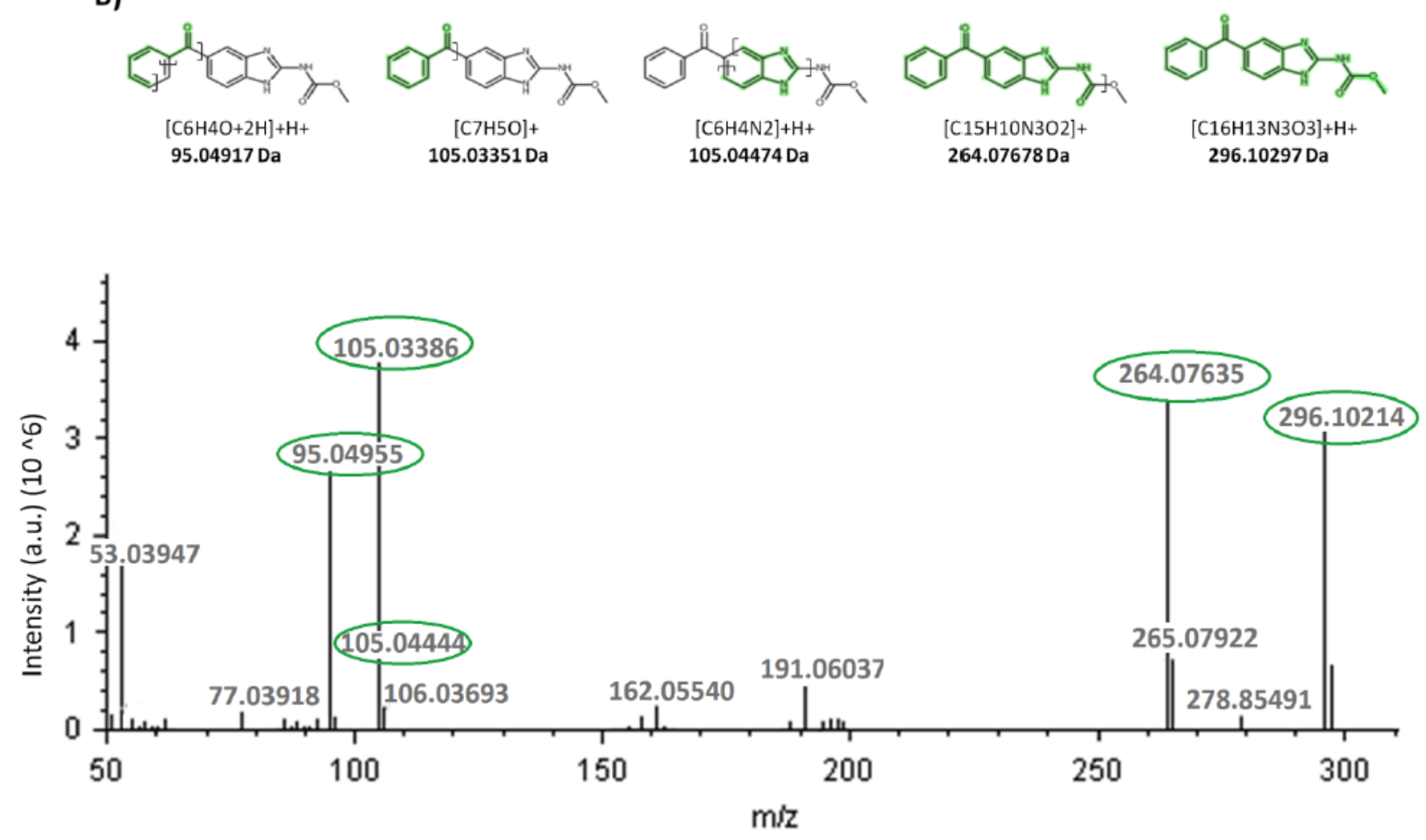
C)
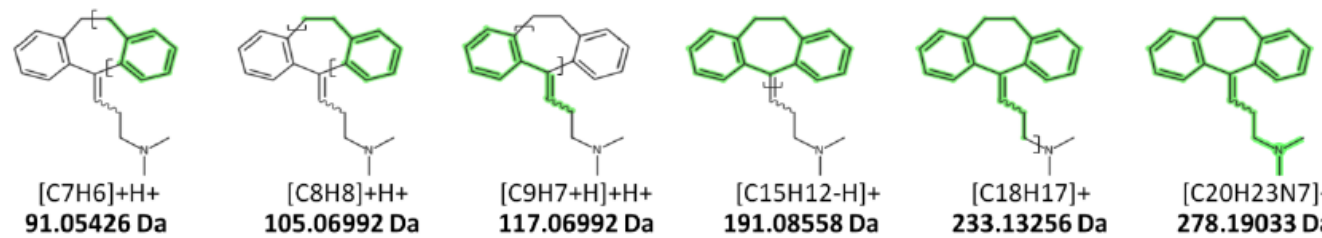

233.13256 Da

[C2OH23N7]+

$278.19033 \mathrm{Da}$

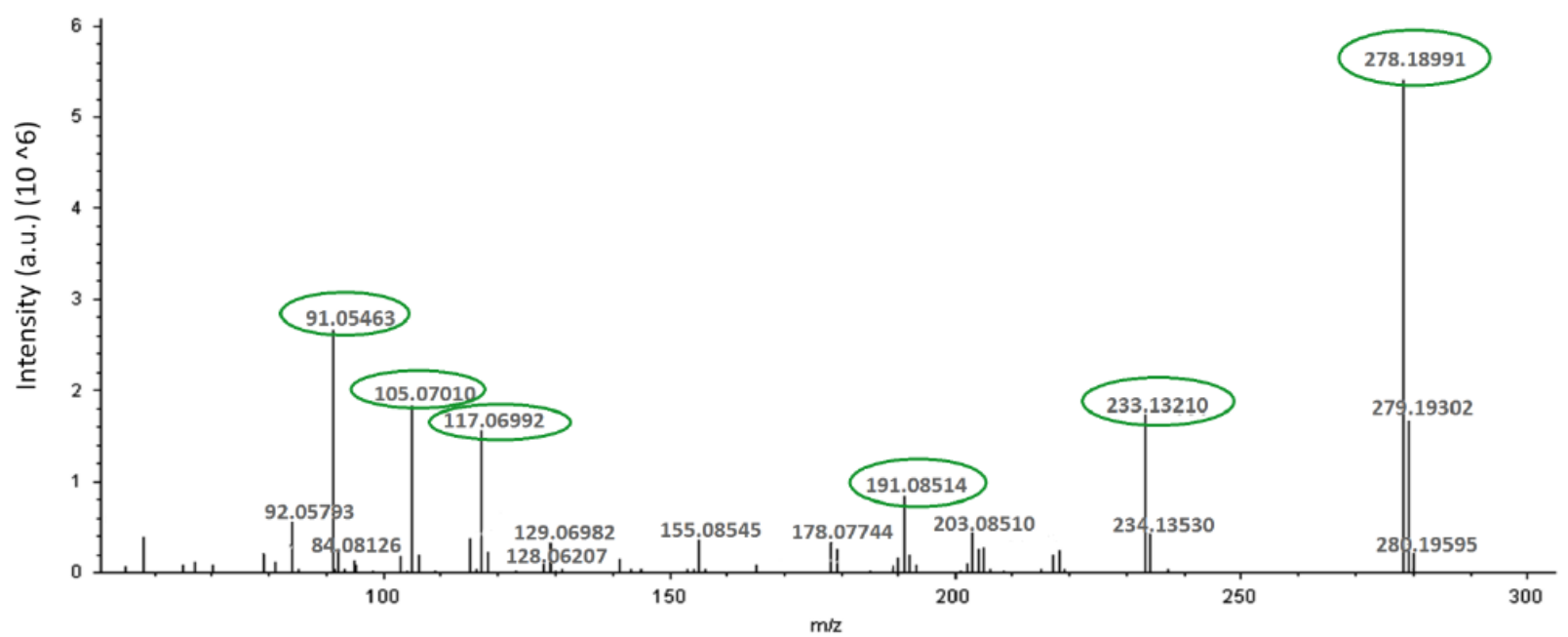

D)
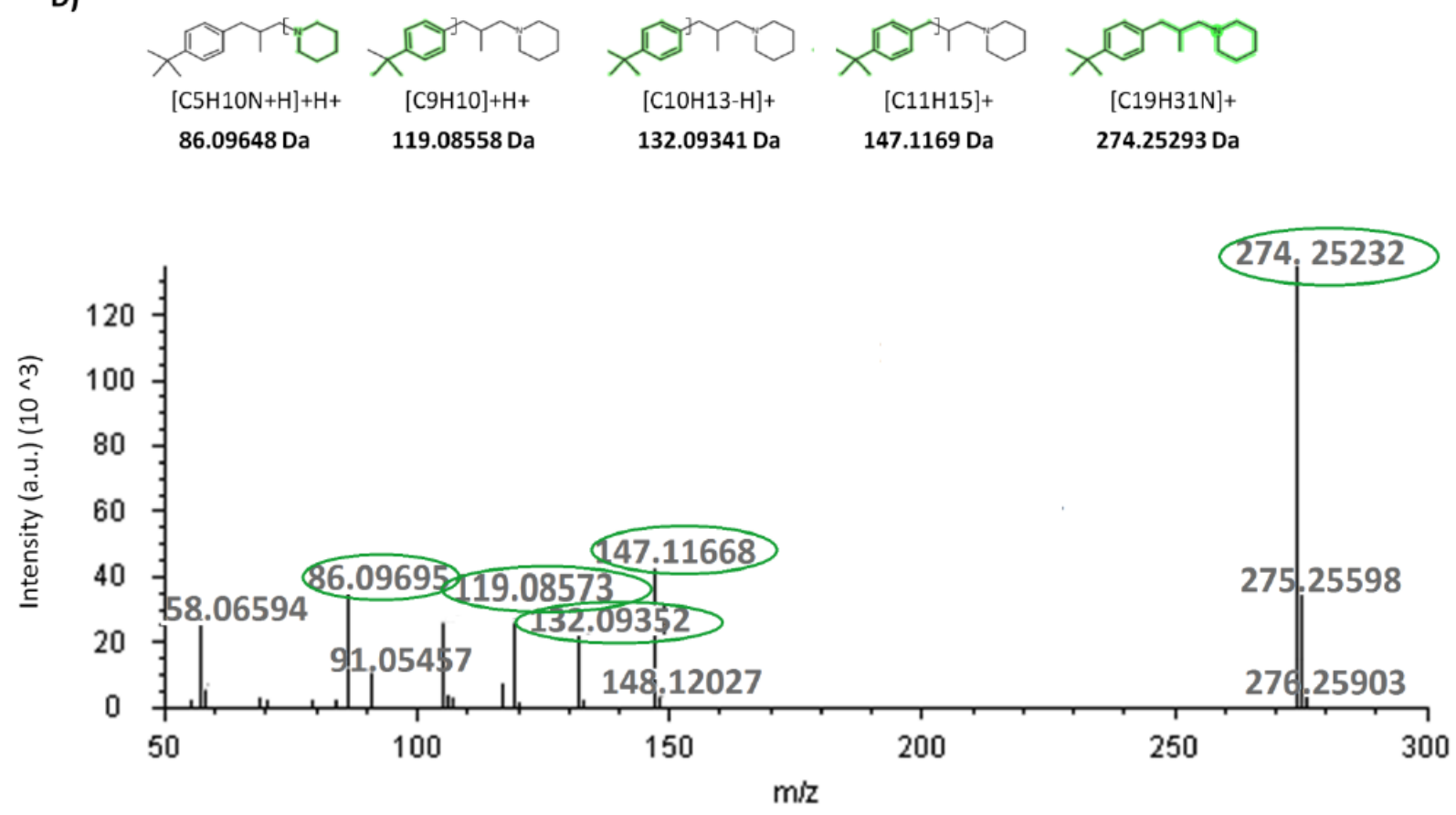
E)<smiles>Cc1cc(CCc2ccc3c(c2)CCC3)cc(CCc2cccc3ccccc23)c1</smiles>

[C4H8N]+ $70.06516 \mathrm{Da}$

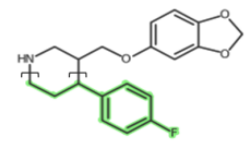

[C9H9F-H]+ $135.06049 \mathrm{Da}$

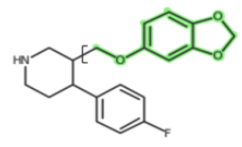

[C8H7O3]+ 151.03899 Da

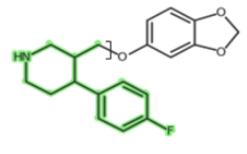

[C12H15FN]+ 192.11837 Da

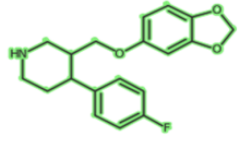

[C19H2OFNO3]+

$330.15000 \mathrm{Da}$

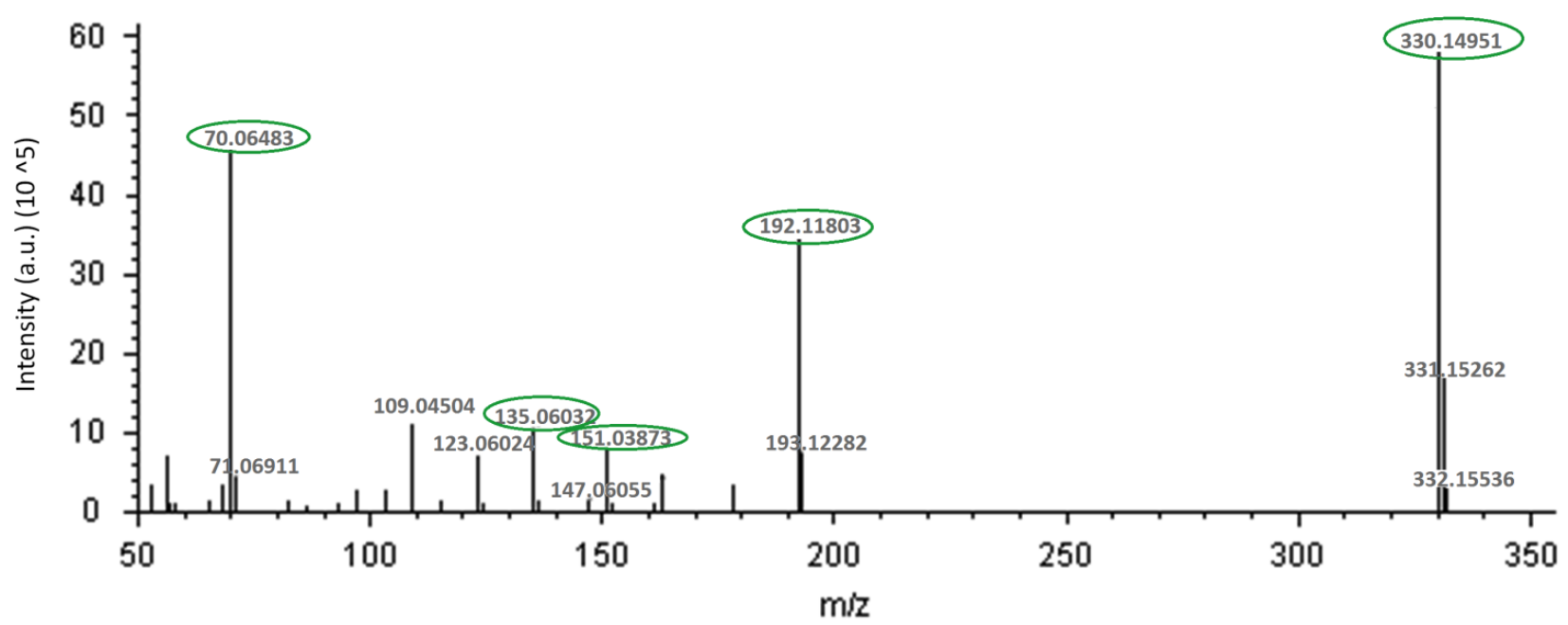

Experimental spectra
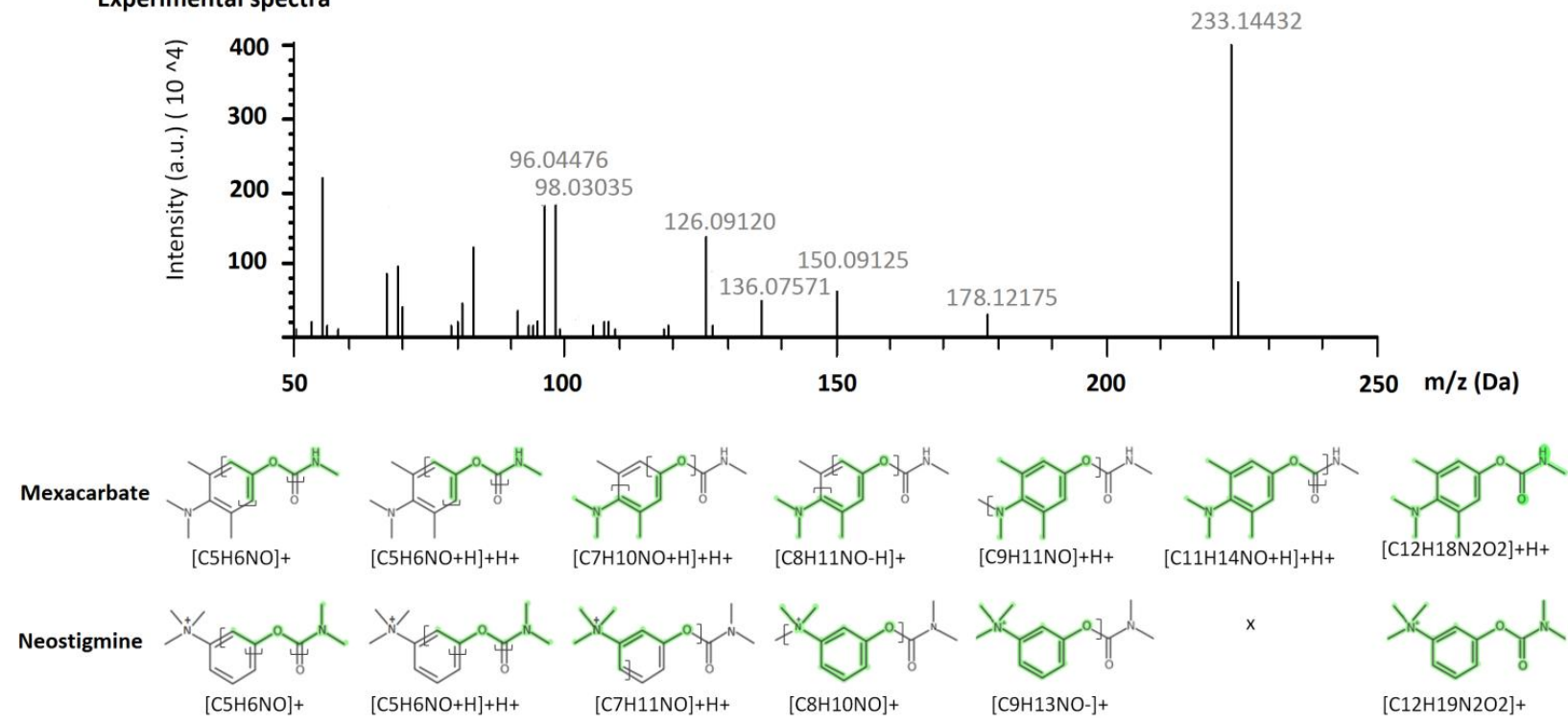

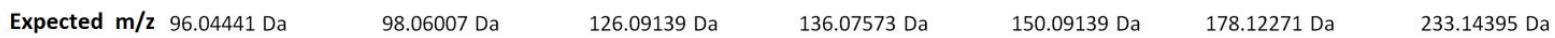

Figure S5 MS $^{2}$ spectra (HCD 10, 35 and 75) of a) albendazole, b) mebendazole, c) amitriptyline, d) fenpropidin e) paroxetine and d) fragments explanation of two potential candidates (mexacarbate and neostigmine) which match with the precursor ion. Only the mayor fragments have been included (rounded). 

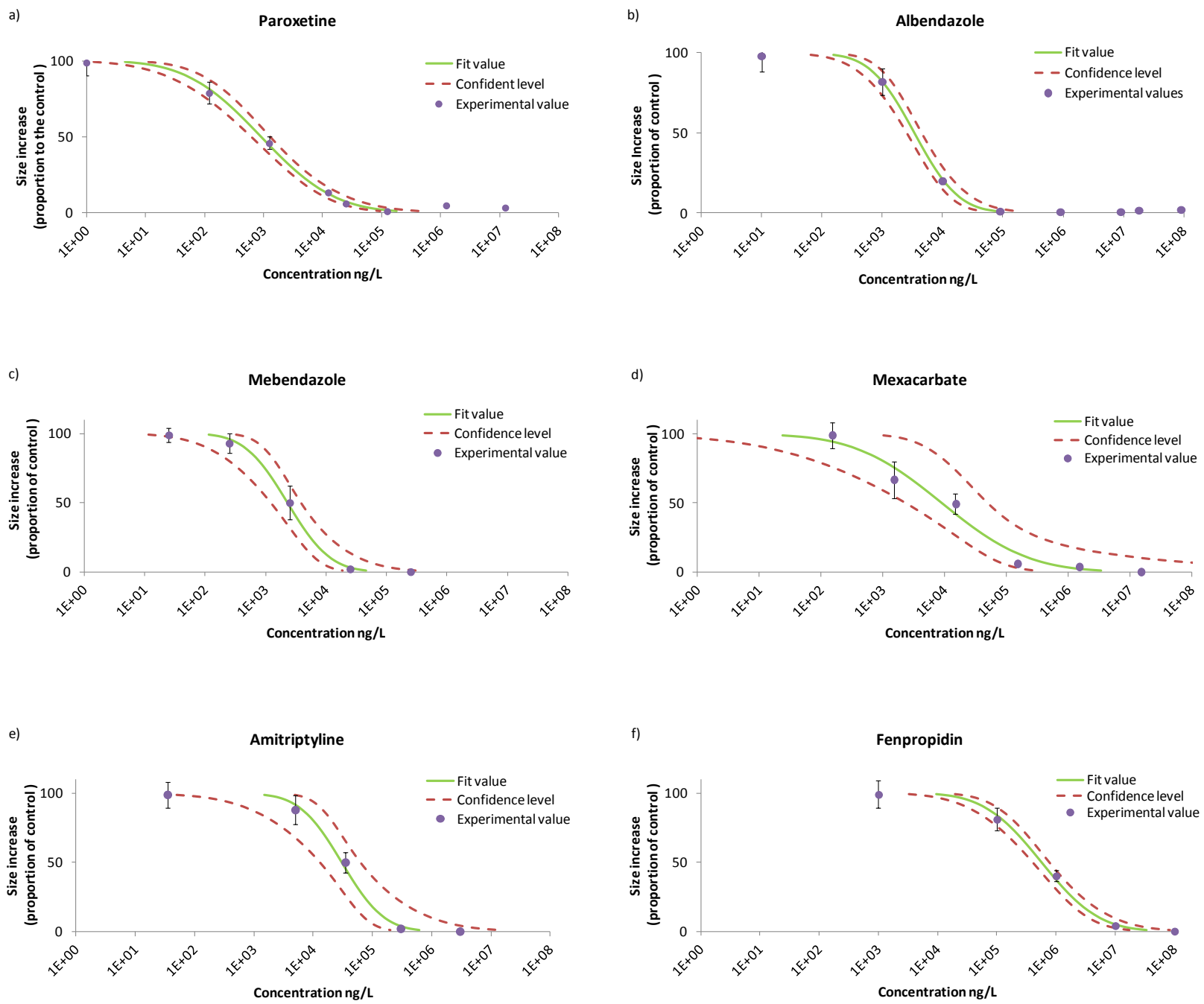

Figure S6 The log dose-response curves of the identified chemicals tested individually: a) paroxetine, b) albendazole, c) mebendazole, d) mexacarbate e) amitriptyline and f) fenpropidin. Straight lines show the EC fit values obtained with Probit and dashed line the confidence level (95\%). 
Table S5. Concentrations of each of the 6 targeted compounds (mexacarbate, albendazole, mebendazole, paroxetine, amitriptyline and fenpropidin) of the artificial mixture at each of the tested relative enrichment factor (REF) dose. Concentrations of REF1 is based on the concentrations presented in the raw sample at equal REF

\begin{tabular}{|c|c|c|c|c|c|c|}
\hline \multicolumn{7}{|c|}{ Concentration (ng/L) } \\
\hline & REF 1 & REF 5 & REF 10 & REF 50 & REF100 & REF1000 \\
\hline Mexacarbate & 17 & 85 & 170 & 850 & 1700 & 17000 \\
\hline Albendazole & 48 & 240 & 480 & 2400 & 4800 & 48000 \\
\hline Mebendazole & 65 & 325 & 650 & 3250 & 6500 & 65000 \\
\hline Paroxetine & 26 & 13 & 260 & 1300 & 2600 & 26000 \\
\hline Amitriptyline & 304 & 1520 & 3040 & 15200 & 30400 & 304000 \\
\hline Fenpropidin & 23 & 115 & 230 & 11500 & 2300 & 23000 \\
\hline
\end{tabular}

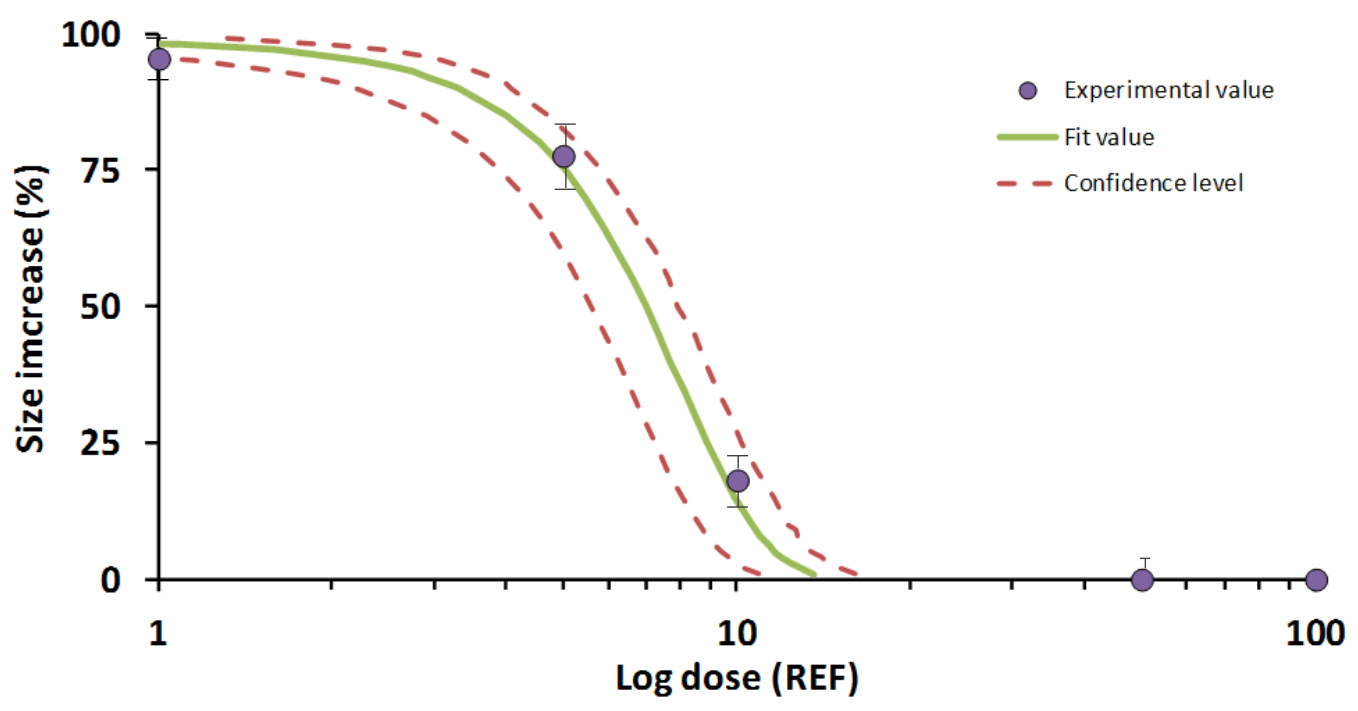

Figure S7. The log dose-response curves of the artificial mixture of the identified 6 chemicals tested all together. Straight lines show the EC fit values obtained with Probit and dashed line the confidence level (95\%). 


\section{S7. References}

(1) Carballeira, C.; Ramos-Gómez, J.; Martín-Díaz, L.; DelValls, T. A. Identification of Specific Malformations of Sea Urchin Larvae for Toxicity Assessment: Application to Marine Pisciculture Effluents. Mar. Environ. Res. 2012, 77, 12-22.

(2) Fernández, N.; Beiras, R. Combined Toxicity of Dissolved Mercury With Copper, Lead and Cadmium on Embryogenesis and Early Larval Growth of the Paracentrotus Lividus Sea-Urchin. Ecotoxicology 2001, 10 (5), 263-271.

(3) Sheridan, R. P.; Simms, M. A. Effect of the Insecticide Zectran (Mexacarbate) on Several Algae. Bull. Environ. Contam. Toxicol. 1975, 13 (5), 565-569..

(4) Parker, B. L.; Dewey, J. E.; Bache, C. A. Carbamate Bioassay Using Daphnia Magna. J. Econ. Entomol. 1970, 63 (3), 710-714.

(5) Beckers, L.-M.; Busch, W.; Krauss, M.; Schulze, T.; Brack, W. Characterization and Risk Assessment of Seasonal and Weather Dynamics in Organic Pollutant Mixtures from Discharge of a Separate Sewer System. Water Res. 2018, 135, 122-133.

(6) Henry, T. B.; Kwon, J.-W.; Armbrust, K. L.; Black, M. C. Acute and Chronic Toxicity of Five Selective Serotonin Reuptake Inhibitors in Ceriodaphnia Dubia. Environ. Toxicol. Chem. 2004, 23 (9), 22292233.

(7) Cunningham, V. L.; Constable, D. J. C.; Hannah, R. E. Environmental Risk Assessment of Paroxetine. Environ. Sci. Technol. 2004, 38 (12), 3351-3359.

(8) Zhang, X. P.; Li, W. X.; Ai, T. S.; Zou, H.; Wu, S. G.; Wang, G. T. The Efficacy of Four Common Anthelmintic Drugs and Traditional Chinese Medicinal Plant Extracts to Control Dactylogyrus Vastator (Monogenea). Aquaculture 2014, 420-421, 302-307.

(9) Rico, A.; Van den Brink, P. J. Probabilistic Risk Assessment of Veterinary Medicines Applied to Four Major Aquaculture Species Produced in Asia. Sci. Total Environ. 2014, 468-469, 630-641. https://doi.org/10.1016/j.scitotenv.2013.08.063.

(10) Oh, S. J.; Park, J.;TLee, M. J.; Park, S. Y.; Lee, J.-H.; Choi, K. Ecological Hazard Assessment of Major Veterinary Benzimidazoles: Acute and Chronic Toxicities to Aquatic Microbes and Invertebrates. Environ. Toxicol. Chem. 2006, 25 (8), 2221-2226. https://doi.org/10.1897/05-493R.1.

(11) Carlsson, G.; Patring, J.; Kreuger, J.; Norrgren, L.; Oskarsson, A. Toxicity of 15 Veterinary Pharmaceuticals in Zebrafish (Danio Rerio) Embryos. Aquat. Toxicol. 2013, 126, 30-41. https://doi.org/10.1016/j.aquatox.2012.10.008. 